\title{
Combined application of Rho-ROCKII and GSK-3 $\beta$ inhibitors exerts an improved protective effect on axonal regeneration in rats with spinal cord injury
}

\author{
GE ZHANG $^{1}$, FEI LEI ${ }^{2}$, QINGZHONG ZHOU ${ }^{2}$, DAXIONG FENG ${ }^{2}$ and YONGHENG BAI ${ }^{3}$ \\ ${ }^{1}$ Department of Orthopedics, The Luzhou People's Hospital; ${ }^{2}$ Department of Spinal Surgery, \\ The First Affiliated Hospital of Luzhou Medical College, Luzhou, Sichuan 646000; ${ }^{3}$ Key Laboratory of Surgery, \\ The First Affiliated Hospital of Wenzhou Medical University, Wenzhou, Zhejiang 325000, P.R. China
}

Received October 9, 2015; Accepted October 17, 2016

DOI: $10.3892 / \mathrm{mmr} .2016 .5918$

\begin{abstract}
Previousstudieshavereported that theRho-associated coiled-coil containing protein kinase 2 (ROCKII) and glycogen synthase kinase- $3 \beta$ (GSK)-3 $\beta$ signaling pathways are involved in axonal regeneration. The present study investigated the effects of the combined application of Y27632 (a ROCKII inhibitor) and 4-benzyl-2-methyl-1,2,4-thiadiazolidine-3,5-dione (TDZD-8; a GSK-3 $\beta$ inhibitor) on neurite outgrowth and functional recovery in rats with spinal cord injury (SCI). A total of 90 female Sprague-Dawley rats were randomly allocated into six groups, and the SCI rats received daily administration of $1.6 \mathrm{mg} / \mathrm{kg}$ Y27632 for 2 weeks and/or $1 \mathrm{mg} / \mathrm{kg}$ TDZD-8 for 3 weeks via a catheter. Cellular apoptosis in the injured spinal cords was measured at each time point using a terminal deoxynucleotidyl transferase-mediated dUTP nick end labeling assay. The expression levels of growth-associated protein-43 (GAP-43) were determined by immunohistochemical staining. In addition, an anterograde tracer was used to analyze axonal regeneration, the Basso Beattie Bresnahan locomotor rating scale (BBB) was analyzed, and the somatosensory evoked potential (SEP) test was conducted. The results demonstrated that SCI upregulated the number of apoptotic cells, increased GAP-43 expression and enhanced the latent periods of SEP, as compared with in mice that underwent a sham operation. Furthermore, SCI decreased the BBB scores and the SEP amplitudes. These
\end{abstract}

Correspondence to: Dr Daxiong Feng, Department of Spinal Surgery, The First Affiliated Hospital of Luzhou Medical College, 25 Taiping Street, Luzhou, Sichuan 646000, P.R. China

E-mail: fdxlz2002@163.com

Dr Yongheng Bai, Key Laboratory of Surgery, The First Affiliated Hospital of Wenzhou Medical University, 2 Fuxue Lane, Wenzhou, Zhejiang 325000, P.R. China

E-mail: baiyongheng@hotmail.com

Key words: spinal cord injury, Rho-ROCKII signaling, Y27632, GSK-3 $\beta$ signaling, TDZD-8, axonal regeneration injuries in the spinal cord were reduced following treatment with Y27632, TDZD-8, or their combined application, as detected by decreased apoptosis, the induction of axonal regeneration, and the promotion of functional recovery of the lower limbs. Although the BBB scores, and SEP amplitudes and latent periods were not significantly different among the three drug treatment groups, the combined application of Y27632 and TDZD-8 resulted in stronger axonal regenerative potency and a greater protective effect on secondary SCI. These results indicated that the combined application of Y27632 and TDZD-8 may more effectively protect against secondary SCI by inhibiting cellular apoptosis, enhancing GAP-43 expression and promoting neurite outgrowth in SCI rats, compared with Y27632 or TDZD-8 alone.

\section{Introduction}

Following injury, axons in the central nervous system (CNS) fail to regenerate (1). Conversely, peripheral nervous system (PNS) axons regenerate following injury and exhibit restored function. This lack of regeneration in the CNS after injury may be associated with the aberrant expression of specific molecules in the CNS myelin and in the glial scar, including Nogo, oligodendrocyte-myelin glycoprotein and myelin-associated glycoprotein (2-4). Previous studies have reported that these molecules induce the activity of the Rho-Rho-associated coiled-coil containing protein kinase 2 (ROCKII) and glycogen synthase kinase-3 $\beta$ (GSK-3 $\beta$ ) signaling pathways, resulting in inhibition of axonal regeneration in the CNS $(5,6)$. Therefore, the Rho-ROCKII and/or GSK-3 $\beta$ signaling pathways may be targeted in order to recover the regenerative ability of axons.

Rho A and ROCKII belong to the AGC [protein kinase (PK)A/PKG/PKC] family of serine/threonine kinases, and are involved in the reorganization of actin cytoskeletal dynamics (7). Two forms of Rho A have been detected in vivo: Rho-GTP (active form) and Rho-GDP (inactive form). Activated Rho A enhances the activity of ROCKII, thus inhibiting axon growth in the CNS $(8,9)$. The upregulation of ROCK activity leads to phosphorylation of various target proteins, including collapsin response mediator 
protein-2 (CRMP-2), a neuronal protein that serves a role in semaphorin-3A-mediated axon guidance during the development of the nervous system (10). Therefore, ROCKII is considered an integration point for regulating actin-myosin contractility and axonal regeneration. Similar to ROCKII, GSK-3 is an active protein kinase that acts as a negative regulator in the hormonal control of glucose homeostasis, and the regulation of transcription factors and microtubules. As a subtype of GSK-3, GSK-3 $\beta$ is highly expressed in neurons during neurite remodeling, and is critical for the establishment of neuronal polarity and development of axons from mature neurites in CNS neurons (11). Overactivity of GSK-3 $\beta$ affects several protein substrates, thus inhibiting the polymerization of microtubules and transport of proteins in the growth cone, and regulating the dynamic balance of microtubules and microfilaments (12). As a result, activated GSK-3 $\beta$ signaling inhibits axonal regeneration.

Previous studies have supported the hypothesis that Rho-ROCKII or GSK-3 $\beta$ inhibitors may improve axon growth following spinal cord injury (SCI) $(13,14)$. Lingor et al reported that inhibition of ROCKII with the small molecule antagonist Y27632 increased neurite outgrowth on chondroitin sulfate proteoglycan in vitro and axonal regeneration in the adult optic nerve in vivo (15). Furthermore, Chan et al suggested that Y27632 exerts beneficial effects on axonal sprouting and functional recovery following rat SCI (16). In addition to Y27632, the selective GSK-3 $\beta$ inhibitor 4-benzyl-2-methyl-1,2,4-thiadiazolidine-3,5-dione (TDZD-8) is also regarded as an important protective factor after SCI. TDZD-8 reduces the development of inflammation and tissue injury, which is associated with spinal cord trauma (17). However, Y27632 or TDZD-8 alone only inhibits one of the signaling pathways involved in protection after SCI. Furthermore, although high doses of Y27632 are beneficial, a low dose is detrimental (18). Therefore, it may be hypothesized that the combined application of Y27632 and TDZD-8 may provide better protection.

The present study investigated the effects of the combined application of Y27632 and TDZD-8 on neurite outgrowth and functional recovery in SCI rats. The results indicated that the combined application of these two inhibitors more effectively protects against secondary SCI by inhibiting cellular apoptosis, enhancing growth-associated protein-43 (GAP-43) expression and promoting neurite outgrowth in SCI rats, compared with Y27632 or TDZD-8 alone.

\section{Materials and methods}

Rats and SCI. A total of 90 female Sprague-Dawley rats (age, 6-8 weeks; weight, 200-250 g) were purchased from the Experimental Animal Center of Luzhou Medical College (Luzhou, China). The rats were housed in a temperature (22-25 $\left.{ }^{\circ} \mathrm{C}\right)$-, humidity (40-60\%)- and light (12-h light/dark cycle)-controlled environment, and were fed standard rat chow and water, this access was controlled. The rats were fasted on the day prior to the experiments. After being anesthetized with pentobarbital sodium $(45-60 \mathrm{mg} / \mathrm{kg}$ ), a surgical longitudinal incision was made along the midline of the back. The spinal cord was exposed using a three-level T9-T11 laminectomy, and SCI was produced by dropping a weight at the T10 level.
Sham-operated rats were subjected to the laminectomy only. All the animals were anesthetized by an intraperitoneal injection with $2 \%$ sodium pentobarbital. In all animals, the L4 segmental spinal cord was exposed and a $3 \mathrm{~cm}$ long epidural catheter was implanted into the spinal dura mater at $\sim 5 \mathrm{~mm}$. The catheter was fixed on the paraspinal muscles and the muscle and skin were sutured. The rats were then housed individually in a temperature-controlled room $\left(25^{\circ} \mathrm{C}\right)$. Paralysis of the lower limbs in rats was used to confirm successful establishment of an SCI model. A total of 1 hour after surgery, the SCI rats began to receive daily doses of Y27632 $(1.6 \mathrm{mg} / \mathrm{kg} / \mathrm{d}$; Sigma-Aldrich; Merck Millipore, Darmstadt, Germany) for 2 weeks and/or TDZD-8 (1 mg/kg/d; Sigma-Aldrich; Merck Millipore) for 3 weeks via a catheter. Rats were sacrificed by cervical dislocation under anesthesia with $0.2 \%$ sodium pentobarbital at various time points, and the injured spinal cord tissues from each SCI rat were fixed in $4 \%$ paraformaldehyde solution.

In the present study, rats were randomly assigned to the following groups $(n=15)$ : i) SCI + Y27632 group, SCI rats were treated with Y27632; ii) SCI + TDZD-8 group, SCI rats were treated with TDZD-8; iii) SCI + TDZD-8 + Y27632 group, SCI rats were treated with TDZD-8 and Y27632; iv) SCI + PBS: SCI rats were treated with $0.01 \%$ PBS; v) SCI group, untreated SCI rats; and vi) sham group, rats were subjected to laminectomy only.

The animal study protocols were approved by the Institutional Animal Care and Use Committee of Luzhou Medical College.

Terminal deoxynucleotidyl transferase-mediated dUTP nick end labeling (TUNEL) assay. A TUNEL assay was performed using In Situ Cell Death Detection kit, POD (Roche Diagnostics, Basel, Switzerland) according to the manufacturer's protocols. The TUNEL assay was conducted on $4-\mu \mathrm{m}$ thick sections, which were dewaxed with xylene and rehydrated with graded ethanol $(100 \%, 95 \%, 85 \%$, and $75 \%)$ and distilled water. The sections were incubated with $10-20 \mu \mathrm{g} / \mathrm{ml}$ proteinase $\mathrm{K}$ for $15 \mathrm{~min}$ at room temperature, and endogenous peroxidase was blocked with $3 \%$ hydrogen peroxide at room temperature for $20 \mathrm{~min}$. The sections were then immersed in terminal deoxynucleotidyl transferase buffer containing deoxynucleotidyl transferase and biotinylated dUTP in a humidified atmosphere at $37^{\circ} \mathrm{C}$ for $90 \mathrm{~min}$. Subsequently, the sections were incubated with a horseradish peroxidase-conjugated antibody (supplied in the TUNEL detection kit) at room temperature for $30 \mathrm{~min}$. The signals were visualized using diaminobenzidine (17) and a DM4000 B LED microscope (Leica Microsystems $\mathrm{GmbH}$, Wetzlar, Germany) and a microscope camera (Leica Microsystems $\mathrm{GmbH}$ ).

Immunohistochemical analysis. Frozen sections (4- $\mu$ m thick) of the injured rat spinal cords were prepared following dehydration in graded sucrose-based solution. The sections were then incubated with a primary antibody targeting GAP-43 (1:500; Epitomics, Burlingame, CA, USA; cat. no. 2256-1) at $4^{\circ} \mathrm{C}$ overnight. Subsequently, the sections were incubated with biotinylated secondary antibody (1:2,000; OriGene Technologies, Inc., Beijing, China; cat. no. SP-9001) at $37^{\circ} \mathrm{C}$ for $30 \mathrm{~min}$ and HRP-labeled streptavidin (OriGene Technologies, 
Inc.) at $37^{\circ} \mathrm{C}$ for $30 \mathrm{~min}$. Diaminobenzidine was used as the chromogen and sections were observed with a DM4000 B LED microscope and microscope camera. Staining was quantified by measuring the intensity of signals using Image-Pro Plus (version 6.0; Media Cybernetics, Inc., Rockville, MD, USA). Two independent investigators semi-quantitatively or quantitatively assessed the immunohistochemical studies in a blinded manner.

Anterograde tracer. A total of 6 weeks after the SCI operation rats were anesthetized with $2 \%$ sodium pentobarbital and a green fluorescent tracer $(<50 \mathrm{mmol} / \mathrm{ml}$; Sigma-Aldrich; Merck Millipore) was injected into the cerebral cortex. After 2 weeks, the SCI rats were sacrificed. Frozen sections $(4-\mu \mathrm{m}$ thick) of the injured spinal cord were prepared, and the staining was quantified by measuring the intensity of signals using Image-Pro Plus (Media Cybernetics, Inc.).

Basso Beattie Bresnahan locomotor rating scale (BBB) score. Motor functions of the lower limbs of the rats were evaluated 3, 6 and 8 weeks after the SCI operation, according to a previous report (19). The motor functions of the lower limbs can be divided into 22 grades. According to function, 0 points indicate hind leg paralysis, whereas 21 indicates completely normal function. BBB scoring was used to assess recovery following contusion injuries to the spinal cord in the rat according to a previous study (20). The BBB score observation period lasted for $4 \mathrm{~min}$, during which the animals should be maintained in the center of the range area.

Somatosensory evoked potential (SEP) monitoring. Spinal SEP monitoring (Nihon Kohden Corporation, Tokyo, Japan) is widely used intraoperatively, due to its ease of use and reliability. In the present study, SEP values were assessed 3 and 8 weeks after the SCI operation, according to a previous report (21). The amplitudes and latent periods of SEP at the $\mathrm{C} 3 / \mathrm{C} 4$ position were recorded by percutaneous electrical stimulation of the contralateral limb of the median nerve and posterior tibial nerve.

Statistical analysis. Data are presented as the mean \pm standard error of the mean. All statistical analyses were performed using SPSS (version 16.0; SPSS Inc., Chicago, USA). A one-way analysis of variance was used when more than two groups were compared followed by Dunnett's method for multiple comparisons. $\mathrm{P}<0.05$ was considered to indicate a statistically significant difference.

\section{Results}

Effects of Rho-ROCKII and GSK-3 $\beta$ inhibitors on cellular apoptosis after SCI. To investigate the combined effects of Rho-ROCKII and GSK-3 $\beta$ inhibitors on cellular apoptosis after SCI, TUNEL-like staining was measured in the perilesional spinal cord tissues $8,24 \mathrm{~h}$ and 1 week after surgery (Fig. 1). As expected, the number of apoptotic cells was significantly higher in SCI rats compared with in the sham group at these time points. Treatment with Y27632, TDZD-8 or combined treatment lowered the number of apoptotic cells in SCI rats compared with in SCI rats treated with or without
PBS, thus suggesting that Rho-ROCKII and GSK-3 $\beta$ inhibitors may protect spinal cord tissues following SCI in rats. In addition, there was no significant difference between the Y27632-treated and TDZD-8-treated groups, which indicated that inhibiting the Rho-ROCKII or GSK-3 $\beta$ pathway exerts similar effects on cellular apoptosis. Combined application of these two inhibitors decreased the number of apoptotic cells compared with Y27632 or TDZD-8 treatment alone 8 and $24 \mathrm{~h}$ after surgery. However, there was no significant difference among these three groups 1 week after SCI. Therefore, it may be hypothesized that the time-dependent downregulatory effect of Y27632, TDZD-8 or their combined application on the number of apoptotic cells in SCI rats may be associated with the body's own natural healing mechanisms, since the reduction in the number of apoptotic cells in SCI rats did not differ among the Y27632-, TDZD-8-, PBS-, Y27632 and TDZD-8-, or untreated groups after 1 week.

Effects of Rho-ROCKII and GSK-3 $\beta$ inhibitors on GAP-43 expression. GAP-43 is a rapidly transported growth-associated protein enriched in elongating axons, which is considered an important endogenous indicator of nerve regeneration $(18,22)$. In the present study, it was demonstrated that the expression of GAP-43 gradually increased in SCI rats over time, as indicated by immunohistochemical staining (Fig. 2). The expression of GAP-43 in SCI rats was significantly higher compared with in sham-operated rats 8 , $24 \mathrm{~h}$ and 1 week after surgery, thus suggesting that injuries to the spinal cord induced marked axonal regeneration. In addition, the expression levels of GAP-43 were higher in SCI rats treated with Y27632, TDZD-8 or combined treatment, as compared with in untreated or PBS-treated SCI rats. These results indicate that Rho-ROCKII and GSK-3 $\beta$ inhibitors may improve axonal regeneration after SCI. Although no significant difference was detected among these three groups 8 and $24 \mathrm{~h}$ after SCI, combined application promoted axonal regeneration more effectively 1 week after surgery compared with Y27632 or TDZD-8 alone.

Effects of Rho-ROCKII and GSK-3 $\beta$ inhibitors on anterograde tracer transmission. The anterograde tracer method was used to investigate the effects of Rho-ROCKII and GSK-3 $\beta$ inhibitors on axonal regeneration. A green fluorescent tracer $(<50 \mathrm{mmol} / \mathrm{ml})$ was injected into the cerebral cortex 6 weeks after SCI operation in rats; this tracer could be transmitted across the axonal gap. The areas of fluorescence at the lesion were measured using a computer-assisted digital image analysis system (Fig. 3). SCI operation promoted axonal regeneration in rats, as indicated by the larger fluorescent areas compared with in the sham group. In addition, the fluorescent areas were larger in drug-treated SCI rats compared with in untreated SCI rats, thus suggesting that Rho-ROCKII and GSK-3 $\beta$ inhibitors improved axonal regeneration. There was no difference between the single drug-treated groups; however, the combined application of Y27632 and TDZD-8 improved axonal regeneration.

Effects of Rho-ROCKII and GSK-3 $\beta$ inhibitors on motor function. The BBB scores at weeks 3,6 and 8 were used to determine the motor function of the lower limbs of rats. As 


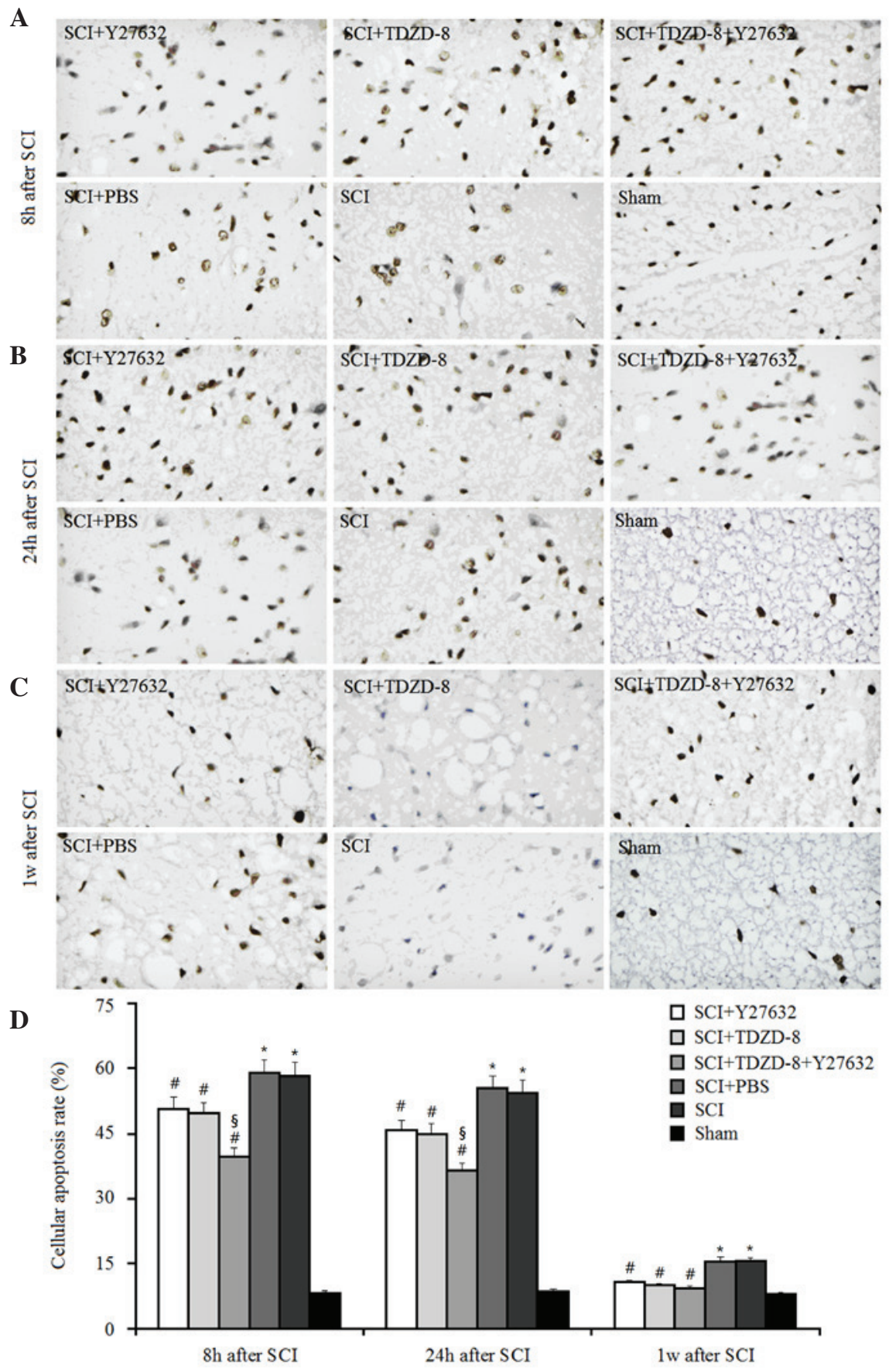

Figure 1. Effects of Rho-Rho-associated coiled-coil containing protein kinase 2 and glycogen synthase kinase-3 $\beta$ inhibitors on cellular apoptosis after SCI. Cellular apoptosis was determined by TUNEL staining in rats following treatment with Y27632, TDZD-8 or their combination for (A) $8 \mathrm{~h}$, (B) $24 \mathrm{~h}$ and (C) 1 week after SCI. Magnification, $\mathrm{x} 400$. (D) Cellular apoptosis rates were analyzed according to the results of TUNEL staining. "P<0.05 vs. sham group; ${ }^{\text {\#P }}<0.05$ vs. SCI group; ${ }^{\S} \mathrm{P}<0.05$ vs. SCI + Y27632 or SCI + TDZD-8 group SCI, spinal cord injury; TDZD-8, 4-benzyl-2-methyl-1,2,4-thiadiazolidine-3,5-dione.

shown in Fig. 4, lower limb function was recovered in SCI rats in a time-dependent manner. The motor function recovered to half its normal level 8 weeks after SCI. Despite the lack of significant differences in the functional recovery of lower limbs among SCI rats with or without drug treatment, Y27632- or TDZD-8-treated rats appeared to exhibit improved recovery.

The present study also examined the latent periods and amplitudes of SEP in SCI rats (Fig. 5). SEP is generated by physiologically or electrically stimulating the afferent peripheral nerve fibers. In SCI rats, downregulated latent periods and upregulated amplitudes of SEP indicate improved functional recovery of spinal cord tissues. The results of the present study indicated that injuries to the spinal cord increased the latent periods of SEP and decreased the amplitudes. In addition, treatment with Y27632 or TDZD-8 inhibited these changes, resulting in a decrease in latent periods and an increase in amplitudes of SEP; however, there were no significant differences between these treatment groups. These findings suggest that treatment with Y27632, TDZD-8 or their combined application exert the same effect on the motor function of the lower limbs of SCI rats. 


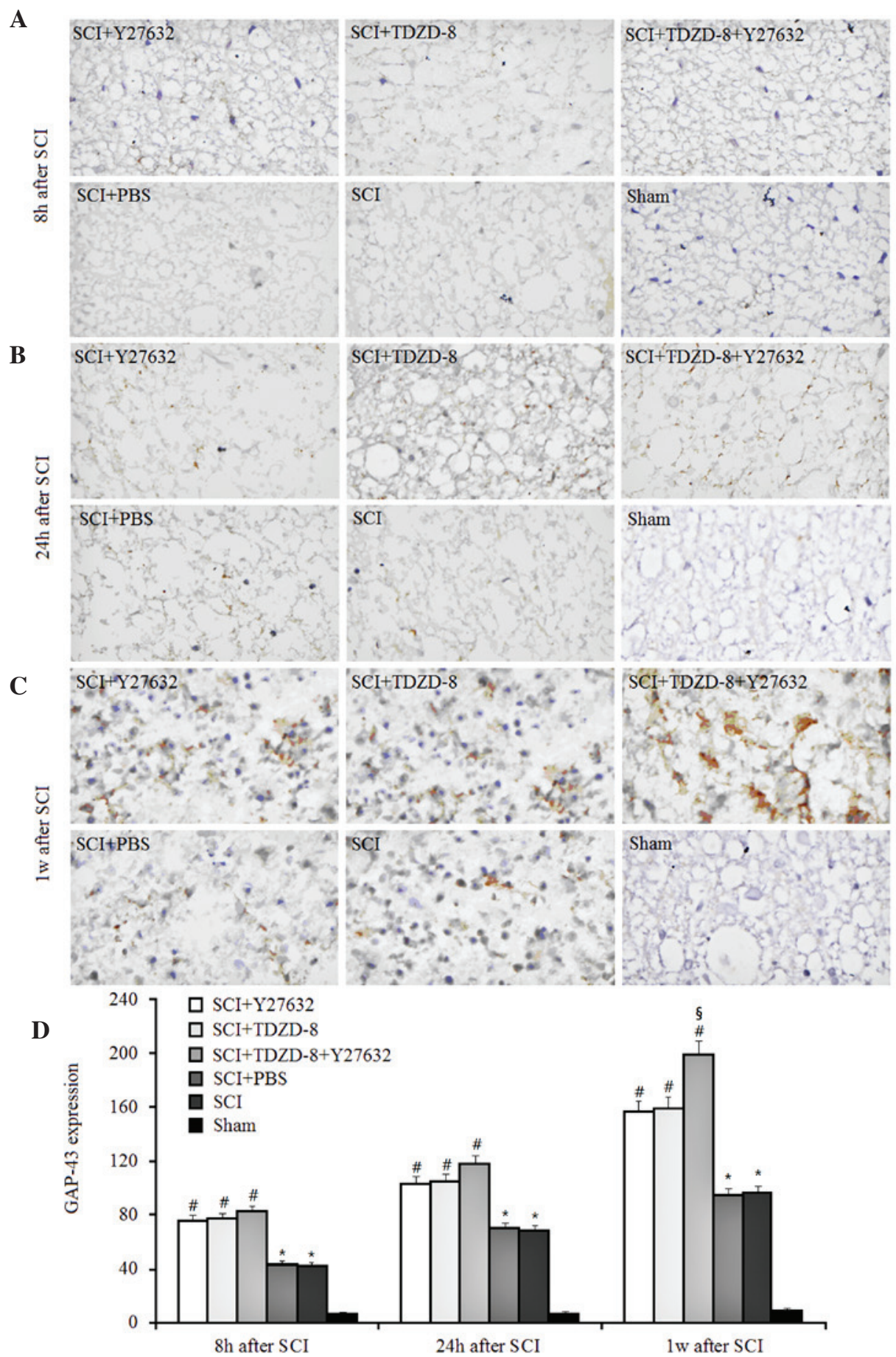

Figure 2. Effects of Rho-Rho-associated coiled-coil containing protein kinase 2 and glycogen synthase kinase-3 3 inhibitors on GAP- 43 expression. Expression of GAP-43 was evaluated by immunohistochemical staining in rats treated with Y27632, TDZD-8 or their combination for (A) $8 \mathrm{~h}$, (B) $24 \mathrm{~h}$ and (C) 1 week after SCI. Magnification, $x 400$. (D) Expression levels of GAP-43 were analyzed according to the results of immunohistochemical staining. "P< $<0.05$ vs. the sham group; "P<0.05 vs. the SCI group; ${ }^{\circledR} \mathrm{P}<0.05$ vs. the SCI + Y27632 or SCI + TDZD-8 group. SCI, spinal cord injury; TDZD-8, 4-benzyl-2-methyl-1,2,4-thiadiazolidine-3,5-dione; GAP-43, growth-associated protein 43.

\section{Discussion}

Effects of Rho-ROCKII inhibitors on axonal regeneration. In the present study, a TUNEL assay and immunohistochemical staining revealed that the number of apoptotic cells was significantly decreased, and the expression of GAP-43 was increased in SCI rats treated with the Rho-ROCKII inhibitor Y27632, as compared with SCI rats treated with or without PBS. In addition, an anterograde tracer analysis indicated that
Y27632 improved axonal regeneration. Furthermore, Y27632 treatment increased BBB scores and SEP amplitudes, and decreased SEP latent periods, thus suggesting that Y27632 protects the motor function of lower limbs in SCI rats. These results indicated that inhibition of the Rho-ROCKII signaling pathway may reduce injuries to the spinal cord and induce marked axonal regeneration in SCI rats.

The protective effects of Rho-ROCKII signaling inhibitors on axonal regeneration reported in the present study are 

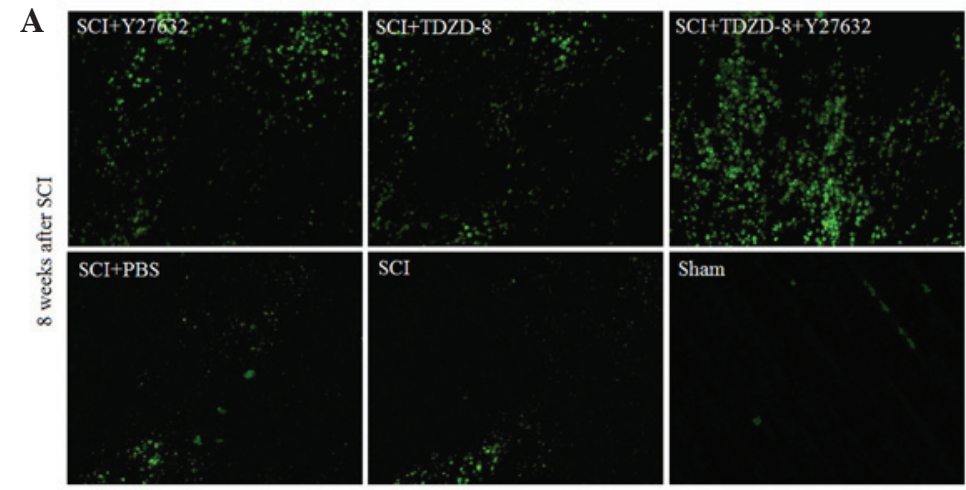

B

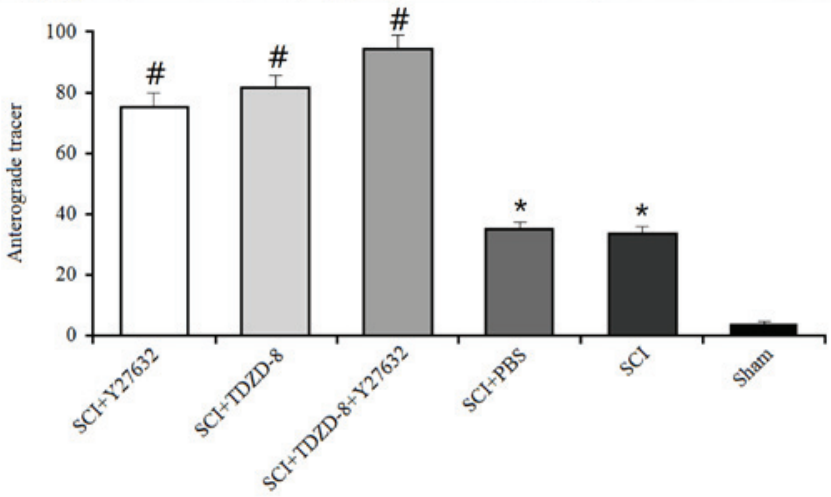

Figure 3. Effects of Rho-Rho-associated coiled-coil containing protein kinase 2 and glycogen synthase kinase-3 $\beta$ inhibitors on anterograde tracer transmission. (A) Axonal regeneration was indicated by the green fluorescent tracer in rats treated with Y27632, TDZD-8 or their combination 8 weeks after SCI. Magnification, $\mathrm{x} 400$. (B) Density of green fluorescence was analyzed using Image-Pro Plus software. ${ }^{*} \mathrm{P}<0.05$ vs. the sham group; ${ }^{\#} \mathrm{P}<0.05$ vs. the SCI group. SCI, spinal cord injury; TDZD-8, 4-benzyl-2-methyl-1,2,4-thiadiazolidine-3,5-dione.

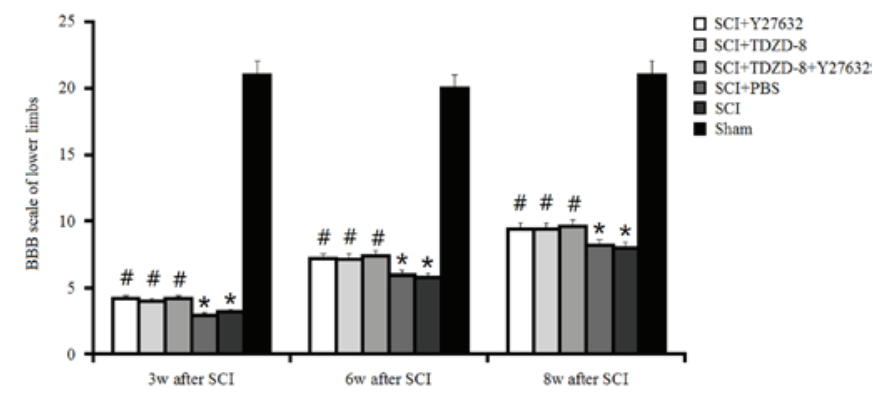

Figure 4. Effects of Rho-associated coiled-coil containing protein kinase 2 and glycogen synthase kinsae-3 $\beta$ inhibitors on BBB scores of lower limbs. The BBB scores of lower limbs were evaluated in rats treated with Y27632, TDZD-8 or their combination 3, 6 and 8 weeks after SCI operation. ${ }^{*} \mathrm{P}<0.05$ vs. the sham group; ${ }^{\text {}} \mathrm{P}<0.05$ vs. the SCI group. SCI, spinal cord injury; TDZD-8, 4-benzyl-2-methyl-1,2,4-thiadiazolidine-3,5-dione; BBB, Basso Beattie Bresnahan locomotor rating scale.

supported by the results of a previous study. Kubo et al demonstrated that suppressing the Rho-ROCKII signaling pathway protects neurons, inhibits apoptosis, reduces glial scars and promotes axonal regeneration (23). Another study by Chan et al also reported that a Rho-ROCKII signaling inhibitor is able to protect axonal sprouting and promote functional recovery after SCI (16). In addition, ROCK inhibition may modulate neurite growth and protect neurons from excitotoxicity-induced cell death (24). These findings suggested that the induction of axonal regeneration by inhibiting Rho-ROCKII signaling is promising.

It is well known that CNS axons do not regenerate as easily as peripheral axons following injury (25), predominantly due to inhibitors present in the CNS myelin and in the glial scar. Myelin-associated inhibitors (MAIs), such as myelin-associated glycoprotein and oligodendrocyte-myelin glycoprotein $(4,26)$, are predominantly expressed at the surface of the cytomembrane, which is adjacent to the axon and myelin sheath. Following SCI, upregulated MAIs activate Rho GTPase and phosphorylate ROCKII (27). The activation of ROCK further leads to phosphorylation of various target proteins, including myosin light chain, CRMP2, and microtubule-associated protein 2 (13). This phosphorylation generates cascading signals, resulting in the breakdown of the growth cone cytoskeleton and inhibition of axonal regeneration. The inhibition of ROCK activity following treatment with Y27632 is able to reduce the injuries to the growth cone cytoskeleton to a certain extent and restore axonal regeneration.

Although Rho-ROCKII signaling inhibitors markedly improved axonal regeneration in the present study, larger in vivo studies are required to identify the appropriate doses of these inhibitors. Chan et al reported that a high dose of Y27632 exerts beneficial effects; however, low doses may be detrimental to rats following SCI (16). In addition, a previous study demonstrated that systemic treatment with high doses of Y27632 significantly enhanced the regeneration of motor axons over short distances, whereas the regeneration of sensory fibers remained largely unchanged (28). Therefore, more studies are required before these inhibitors can be used in clinical practice.

Effects of $G S K-3 \beta$ inhibitors on axonal regeneration. In addition to Rho-ROCKII signaling inhibitors, GSK-3 $\beta$ signaling 


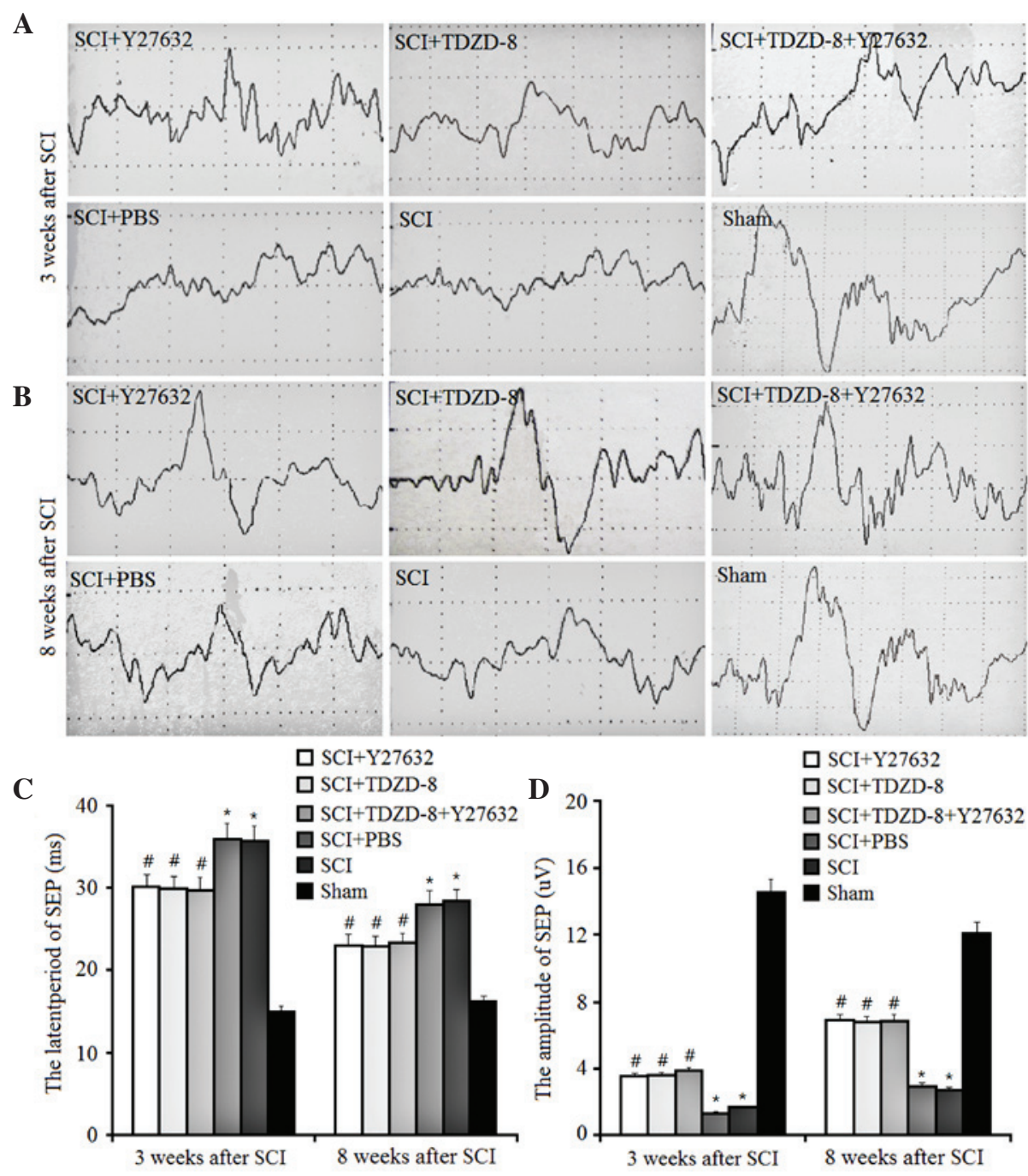

Figure 5. Effects of Rho-Rho-associated coiled-coil containing protein kinase 2 and glycogen synthase kinase-3 $\beta$ inhibitors on SEP. The SEPs were examined in rats treated with Y27632, TDZD-8 or their combination at (A) 3 and (B) 8 weeks after SCI. The SEPs were further analyzed, including the (C) latent periods and (D) amplitudes. "P<0.05 vs. sham group; ${ }^{\text {P }}<0.05$ vs. SCI group. SCI, spinal cord injury; TDZD-8, 4-benzyl-2-methyl-1,2,4-thiadiazolidine-3,5-dione; SEP, somatosensory evoked potential.

inhibitors have been reported to protect axonal regeneration following SCI $(17,29)$. The present study demonstrated that the GSK-3 $\beta$ signaling inhibitor TDZD-8 significantly decreased the number of apoptotic cells, increased GAP-43 expression and induced marked axonal regeneration in SCI rats. In addition, TDZD- 8 administration protected the motor function of the lower limbs of SCI rats, as detected by increased BBB scores and SEP amplitudes, and decreased SEP latent periods. These results indicated that inhibition of GSK-3 $\beta$ signaling may alleviate SCI and promote axonal regeneration.

Previous studies have reported that axon guidance molecules are more abundant following SCI, and neuropilin-1/plexin-A serves to activate phosphatidylinositol-3 kinase and produce phosphatidylinositol 3,4,5-triphosphate. These activated factors further activate GSK-3 $\beta$ and produce a signaling cascade that collapses the growth cone. Kim et al reported that a stronger knockdown of GSK-3 $\beta$ markedly reduced axonal growth in dissociated cultures and slice preparations, whereas a moderate reduction of GSK-3 activity, via the use of pharmacological inhibitors, induced axon branching (14). GSK-3 is a downstream convergent point for several axon growth regulatory pathways. As the first non-competitive inhibitor of ATP, TDZD-8 is more selective than other inhibitors of GSK-3 $\beta$. In addition to the effects of the Rho-ROCKII inhibitor Y27632, the results of the present study demonstrated that treatment with TDZD-8 reduced SCI and induced axonal regeneration in SCI rats. However, the effects of TDZD-8 and Y27632 on axonal regeneration and functional recovery of the lower limbs were not significantly different. It may be hypothesized that this lack of difference is due to differences in sensitivity. In addition, the dose used in the present study may have affected the results; therefore, the appropriate in vivo dosage requires further exploration.

Effects of combined application of Rho-ROCKII and GSK-3 $\beta$ inhibitors on axonal regeneration. Several inhibitors appear in the in vivo microenvironment following SCI; therefore, the effect of suppressing only one of these inhibitors in order to 
promote axonal regeneration is limited. Gopalakrishnan et al suggested that a ROCK inhibitor alone could not completely abolish the inhibitory effects of chondroitin sulfate proteoglycans (30). TDZD-8 has been shown to inhibit activation of GSK-3 $\beta$, leading to CRMP-2 dephosphorylation; however, it was unable to inhibit ROCK-induced phosphorylation (15). Therefore, TDZD-8 could not completely inhibit the collapse of the growth cone. Simultaneous inhibition of ROCKII and GSK-3 $\beta$ signaling may abolish the inhibitory effects of endogenous inhibitors and effectively induce axonal regeneration following SCI. The results of the present study further supported the hypothesis that combined administration of Rho-ROCKII and GSK-3 $\beta$ inhibitors could improve axonal regeneration.

The protective effects of combined application of Rho-ROCKII and GSK-3 $\beta$ inhibitors on axonal regeneration are associated with the reduction of cellular apoptosis. A previous study by Dubreuil et al suggested that Rho is activated after SCI and upregulates the expression of p75 neurotrophin receptor (p75NTR), thus resulting in apoptosis (31). Conversely, suppressing the overactivation of Rho after SCI protects cells from p75NTR-dependent apoptosis. Furthermore, Cuzzocrea et al reported that TDZD-8 was able to inhibit apoptosis by upregulating B-cell lymphoma 2 (Bcl-2) expression and downregulating Bcl-2-associated X protein expression (17). In addition, TDZD-8 could inhibit apoptosis in the hippocampus following ischemia-reperfusion injury by reducing the release of cytochrome $c$ and the activation of caspase-9, which promotes mitochondria-mediated apoptosis (32). These findings indicated that the Rho-ROCKII and GSK-3 $\beta$ signaling pathways are involved in apoptosis following SCI, and the protective effects of suppressing one signaling pathway alone may be limited. The combined application of Rho-ROCKII and GSK-3 $\beta$ inhibitors may yield better results. The present study demonstrated that the number of apoptotic cells was decreased in SCI rats following treatment with Y27632 or TDZD-8. However, the reduction in the number of apoptotic cells during the early stage of SCI was more pronounced after the combined administration of Y27632 and TDZD-8, as compared with Y27632 or TDZD-8 alone. Therefore, the combined administration of Rho-ROCKII and GSK-3 $\beta$ signaling inhibitors may more effectively protect against cellular apoptosis, which may serve an important role in axonal regeneration.

In addition, the functional recovery of lower limbs in rats is an important indicator of axonal regeneration after SCI. However, the BBB and SEP results were not significantly altered in the treatment groups compared with the SCI group. In rats, the SCI operation results in neuronal loss and axonal injury. Treatment with Y27632 or TDZD-8, or their combined administration, could only reduce these injuries in axons and promote axonal regeneration, but could not restore lost neurons. Therefore, the functional recovery of the lower limbs in SCI rats was limited and incomplete, although drug-induced axonal regeneration relieves SCI to a certain extent. Future pharmacological studies should investigate the protection of neuronal loss, in addition to the induction of axonal regeneration.

In conclusion, in the present study, an SCI rat model was administered daily doses of the ROCKII inhibitor Y27632 and/or the GSK-3 $\beta$ inhibitor TDZD-8. Subsequently, the degree of injury in the spinal cord, axonal regeneration and the functional recovery of lower limbs were investigated. The results demonstrated that treatment with Y27632 and TDZD-8 significantly inhibited cellular apoptosis, enhanced GAP-43 expression and promoted neurite outgrowth. In addition, the combined application of Y27632 and TDZD-8 more effectively protected the spinal cords of SCI rats from secondary injuries, when compared with Y27632 or TDZD-8 alone.

\section{Acknowledgements}

The present study was sponsored by the National Natural Science Foundation of China (grant no. Y20110028) and the Project of Sichuan Department of Science and Technology (grant no. 2015JY0224).

\section{References}

1. Horner PJ and Gage FH: Regenerating the damaged central nervous system. Nature 407: 963-970, 2000.

2. Chen MS, Huber AB, van der Haar ME, Frank M, Schnell L, Spillmann AA, Christ F and Schwab ME: Nogo-A is a myelin-associated neurite outgrowth inhibitor and an antigen for monoclonal antibody IN-1. Nature 403: 434-439, 2000.

3. Domeniconi M, Cao Z, Spencer T, Sivasankaran R, Wang K, Nikulina E, Kimura N, Cai H, Deng K, Gao Y, et al: Myelin-associated glycoprotein interacts with the Nogo66 receptor to inhibit neurite outgrowth. Neuron 35: 283-290, 2002.

4. Wang KC, Koprivica V, Kim JA, Sivasankaran R, Guo Y, Neve RL and He Z: Oligodendrocyte-myelin glycoprotein is a nogo receptor ligand that inhibits neurite outgrowth. Nature 417: 941-944, 2002.

5. Madura T, Yamashita T, Kubo T, Fujitani M, Hosokawa K and Tohyama M: Activation of Rho in the injured axons following spinal cord injury. EMBO Rep 5: 412-417, 2004.

6. Alabed YZ, Pool M, Ong Tone S, Sutherland C and Fournier AE: GSK3 beta regulates myelin-dependent axon outgrowth inhibition through CRMP4. J Neurosci 30: 5635-5643, 2010.

7. Schmandke A, Strittmatter SM and Strittmatter SM: ROCK and Rho: Biochemistry and neuronal functions of Rho-associated protein kinases. Neuroscientist 13: 454-469, 2007.

8. Fournier AE, Takizawa BT and Strittmatter SM: Rho kinase inhibition enhances axonal regeneration in the injured CNS. J Neurosci 23: 1416-1423, 2003.

9. Shao Z, Browning JL, Lee X, Scott ML, Shulga-Morskaya S, Allaire N, Thill G, Levesque M, Sah D, McCoy JM, et al: TAJ/TROY, an orphan TNF receptor family member, binds Nogo-66 receptor 1 and regulates axonal regeneration. Neuron 45: 353-359, 2005.

10. Arimura N, Inagaki N, Chihara K, Ménager C, Nakamura N, Amano M, Iwamatsu A, Goshima Y and Kaibuchi K: Phosphorylation of collapsin response mediator protein-2 by Rho-kinase. evidence for two separate signaling pathways for growth cone collapse. J Biol Chem 275: 23973-23980, 2000.

11. Yoshimura T, Kawano Y, Arimura N, Kawabata S, Kikuchi A and Kaibuchi K: GSK-3beta regulates phosphorylation of CRMP-2 and neuronal polarity. Cell 120: 137-149, 2005.

12. Zhou FQ and Snider WD: Cell biology. GSK-3beta and microtubule assembly in axons. Science 308: 211-214, 2005.

13. Mueller BK, Mack H and Teusch N: Rho kinase, a promising drug target for neurological disorders. Nat Rev Drug Discov 4: 387-398, 2005.

14. Kim WY, Zhou FQ, Zhou J, Yokota Y, Wang YM, Yoshimura T, Kaibuchi K, Woodgett JR, Anton ES and Snider WD: Essential roles for GSK-3s and GSK-3-primed substrates in neurotrophin-induced and hippocampal axon growth. Neuron 52: 981-996, 2006.

15. Lingor P, Teusch N, Schwarz K, Mueller R, Mack H, Bähr M and Mueller BK: Inhibition of Rho kinase (ROCK) increases neurite outgrowth on chondroitin sulphate proteoglycan in vitro and axonal regeneration in the adult optic nerve in vivo. J Neurochem 103: 181-189, 2007. 
16. Chan CC, Khodarahmi K, Liu J, Sutherland D, Oschipok LW, Steeves JD and Tetzlaff W: Dose-dependent beneficial and detrimental effects of ROCK inhibitor Y27632 on axonal sprouting and functional recovery after rat spinal cord injury. Exp Neurol 196: 352-364, 2005.

17. Cuzzocrea S, Genovese T, Mazzon E, Crisafulli C, Di Paola R, Muià C, Collin M, Esposito E, Bramanti $\mathrm{P}$ and Thiemermann C: Glycogen synthase kinase-3 beta inhibition reduces secondary damage in experimental spinal cord trauma. J Pharmacol Exp Ther 318: 79-89, 2006.

18. Kawasaki T, Nishio T, Kawaguchi $\mathrm{S}$ and Kurosawa $\mathrm{H}$ : Spatiotemporal distribution of GAP-43 in the developing rat spinal cord: A histological and quantitative immunofluorescence study. Neurosci Res 39: 347-358, 2001.

19. Park DY, Mayle RE, Smith RL, Corcoran-Schwartz I, Kharazi AI and Cheng I: Combined transplantation of human neuronal and mesenchymal stem cells following spinal cord injury. Global Spine J 3: 1-6, 2014

20. Basso DM, Beattie MS and Bresnahan JC: A sensitive and reliable locomotor rating scale for open field testing in rats. J Neurotrauma 12: 1-21, 1995.

21. Caizhong X, Chunlei S, Beibei L, Zhiqing D, Qinneng D and Tong W: The application of somatosensory evoked potentials in spinal cord injury rehabilitation. NeuroRehabilitation 35 835-840, 2014

22. Kim DH and Jahng TA: Continuous brain-derived neurotrophic factor (BDNF) infusion after methylprednisolone treatment in severe spinal cord injury. J Korean Med Sci 19: 113-122, 2004

23. Kubo T, Hata K, Yamaguchi A and Yamashita T: Rho-ROCK inhibitors as emerging strategies to promote nerve regeneration. Curr Pharm Des 13: 2493-2499, 2007.
24. Jeon BT, Jeong EA, Park SY, Son H, Shin HJ, Lee DH, Kim HJ, Kang SS, Cho GJ, Choi WS and Roh GS: The Rho-kinase (ROCK) inhibitor Y-27632 protects against excitotoxicity-induced neuronal death in vivo and in vitro. Neurotox Res 23: 238-248, 2012.

25. Fournier AE and Strittmatter SM: Repulsive factors and axon regeneration in the CNS. Curr Opin Neurobiol 11: 89-94, 2001.

26. Liu BP, Fournier A, GrandPre T and Strittmatter SM: Myelin-associated glycoprotein as a functional ligand for the Nogo-66 receptor. Science 297: 1190-1193, 2002.

27. Buchsbaum RJ: Rho activation at a glance. J Cell Sci 120: 1149-1152, 2007.

28. Joshi AR, Bobylev I, Zhang G, Sheikh KA and Lehmann HC: Inhibition of Rho-kinase differentially affects axon regeneration of peripheral motor and sensory nerves. Exp Neurol 263: 28-38, 2015.

29. Dill J, Wang H, Zhou F and Li S: Inactivation of glycogen synthase kinase 3 promotes axonal growth and recovery in the CNS. J Neurosci 28: 8914-8928, 2008

30. Gopalakrishnan SM, Teusch $\mathrm{N}$, Imhof $\mathrm{C}$, Bakker $\mathrm{MH}$, Schurdak M, Burns DJ and Warrior U: Role of Rho kinase pathway in chondroitin sulfate proteoglycan-mediated inhibition of neurite outgrowth in PC12 cells. J Neurosci Res 86: 2214-2226, 2008.

31. Dubreuil CI, Winton MJ and McKerracher L: Rho activation patterns after spinal cord injury and the role of activated Rho in apoptosis in the central nervous system. J Cell Biol 162: 233-243, 2003

32. Collino M, Thiemermann C, Mastrocola R, Gallicchio M, Benetti E, Miglio G, Castiglia S, Danni O, Murch O, et al: Treatment with the glycogen synthase kinase-3beta inhibitor, TDZD-8, affects transient cerebral ischemia/reperfusion injury in the rat hippocampus. Shock 30: 299-307, 2008. 